\title{
Annonce du diagnostic et rupture biographique
}

\author{
Tinsa Francine \\ Département de sociologie \\ Faculté de Lettres et des Sciences Humaines \\ Universitet de Sfax, Tunisie \\ E-mail: tinsafrancine@yahoo.fr
}

\section{Abstract}

Dans ce papier, nous nous référons à la théorisation ancrée pour approfondir l'un des premiers segments de la trajectoire de la maladie cancéreuse, celui de l'annonce du diagnostic. Notre point de départ est la théorie des contextes de conscience, formulée en 1965 par Strauss et Glaser. Identifiées, durant la phase empirique, comme catégories principales, par une analyse inductive, les différents contextes de l'annonce du diagnostic se sont construits à partir des différentes stratégies d'annonce répertoriées au moment de l'observation, des intentions des professionnels, des attentes livrées par les patients en matière d'information soubaitée, des conditions spécifiques entourant l'action. Les informateurs principaux $(n=21)$ sont des patients atteints d' un cancer et hospitalisés pour une cure de chimiothérapie. D'autres informateurs contextuels ( cliniciens oncologues, bénévoles, parents et proches, cadres hospitaliers...) contribuent également au recueil des données. L'objectif poursuivi est de prendre en compte les motivations des praticiens et le sens qu'ils accordent à l'étape de l'annonce et de les recontextualiser en référence à l'encadrement prévu par l'instance normative. On y discute quelques conditions spécifiques, liées au contexte organisationnel qui freinent le degré d'ouverture et on s' interroge de l' impact de ce manque d' ouverture sur l'entrée dans la maladie ainsi que sur les prochaines étapes de chaque itinéraire thérapeutique

The paper refers to the "grounded theory" (théorisation ancrée) targeting an indepth exploration of the announcement of the diagnosis as one of the first components of the trajectory of cancer. We considered first the theory of the contexts awareness, formulated in 1965 by Strauss and Glaser. The contexts of the announcement of diagnosis have been identified as major categories through an inductive analysis within the empiric phase. They have been built based on different announcement strategies recorded during the observation of the practitioners' intentions, on expectations expressed by patients in term of information, on specific conditions around the actions. The key informants $(n=21)$ are patients having cancer and hospitalized for chemotherapy. Other local informants (clinical oncologists, volunteers, parents and closers, hospital cadres...) 
have contribute to the data collection. The aim is to address the practitioners' motivations and the meaning they give to the step of the announcement of the diagnosis. We also aim at recontextualizing that step according to the tutoring planned by the normative instance. The paper analyses specific conditions linked to the organizational contexts that slow the scope of opening. We are wondering about the impact of such lack of opening and introduction to the disease as well as about the next steps of each therapeutic itinerary.

\section{Introduction}

Le vieillissement de la population, des changements importants au niveau du style de vie aussi bien que des modifications environnementales ou encore des progrès opérés dans le domaine du dépistage et du traitement contribuent à expliquer la progression du cancer en Tunisie, maladie chronique identifiée parmi les plus répandues et les plus mortelles, la première place des cause de décès étant les maladies cardio- vasculaires.

Le cancer reste une pathologie de la personne agée mais il touche également des hommes et des femmes de moins de 65 ans et de jeunes enfants. D'après le Registre du Cancer du Sud Tunisien , paru en l'an 2000 , concernant les années 1997-1999, l'incidence globale standardisée se situerait pour ces années, toutes localisations confondues, à 101,5 cas / 100.000 pour le sexe masculin et 66,0 / 100.000 pour le sexe féminin

Partir du cancer et des orientations stratégiques en matière de prise en charge nous place devant une diversité de choix posés et initiés. Parmi ces choix nous retrouvons la déhospitalisation des malades et par conséquent le déplacement d'une partie du traitement en ambulatoire, le projet d'élargir la surveillance et le soutien thérapeutique aux régions éloignées des centres spécialisés, une réflexion sur la mise en place d'un réseau de soutien et d'accompagnement des malades en fin de vie, dans un pays où la famille témoigne de son sens pratique et de sa volonté d'accompagner un proche devant franchir la dernière étape de sa vie et se trouve par conséquent exposée à des situations de stress, vu la lourdeur des responsabilités et la discontinuité qu' on remarque entre les structures spécialisées et les milieux familiaux .

D'autres aspects tel que la lutte incessante pour rendre la douleur plus tolérable, par l'intermédiaire de la contribution de médecins spécialistes formés à cette approche aussi bien que par l'initiation de médecins diplômés en médecine générale à travailler en relais avec les spécialistes oncologues et bien d'autres projets amorcés ou du moins planifiés se joignent à ceux énumérés précédemment. Tout ceci nous rappelle des orientations actuelles de la politique de santé en cancérologie et plus spécifiquement celles qui concernent le traitement et l'accompagnement des individus touchés par ce type de pathologie. D’abord des efforts répétitifs pour améliorer la qualité de la prise en charge tout en allégeant la structure hospitalière par la réduction des temps de séjour et quelques tentatives de redéploiement des activités des praticiens spécialisés dans le 
domaine de l'oncologie, partant d'un questionnement sur le type de collaboration qui pourrait s'instaurer entre différents partenaires à l' exemple des médecins généralistes.

Ces projets sont initiés à l'intérieur de la sphère médicale et se fondent autant sur des principes éthiques de la pratique que sur des valeurs d'efficience et d'efficacité.

Or on constate que de nombreux problèmes liés étroitement à l' expérience du cancer et à l'image véhiculée à propos de la pathologie continuent d' assombrir les trajectoires de la maladie et interfèrent avec l'atteinte des objectifs visés, en rapport avec le dépistage précoce et les projets d'acheminer et d'individualiser les traitements curatifs requis par l'état de santé du malade, en autant que la disponibilité des produits prescrits en laisse l'opportunité.

Parmi les problèmes identifiés, on retrouve une symbolique morbide très ancrée dans les représentations sociales de la maladie cancéreuse et des institutions spécialisées dans le domaine de l'oncologie. En raison de ces représentations, les relations du malade avec son réseau familial aussi bien qu'avec les autres mondes sociaux dans lesquels il demeure impliqué ou du moins lié, s'en trouvent affectées. Et du côté des professionnels, le non dit ou du moins la difficulté à trouver les mots que 1 on considèrent comme justes et non traumatisants continuent de préoccuper les spécialistes et se répercutent, selon l'opinion des malades, sur la manière de composer avec les différents événements de cette douloureuse expérience.

Cette réalité nous invite à nous interroger sur le cheminement de l' individu pour mettre en relief l' influence du contexte actuel sur la prise de décision en rapport avec toute activité qui pourrait contribuer à optimiser sa santé, lutter conte les risques de maladies ou dans le cas où la maladie se trouve déclarée, participer plus activement à la thérapeutique initiée.

Nous avons donc choisi, pour le présent article, de nous arrêter plus spécifiquement sur le moment de l'entrée dans la maladie au sens de Strauss le définissant comme une révélation pour le «self» et les autres de la transformation de l'identité sociale qui s'accomplit dans une contrainte de cheminement.

Deux enquêtes, celle de Charmaz réalisée auprès de patients touchés par la maladie chronique et celle de Bury, consacrée plus spécifiquement aux individus atteints de polyarthrite rhumatoïde illustrent cette forme de rupture marquant, dans un premier temps, l'entrée dans la maladie. D'abord Charmaz ,1987 se situant dans la lignée de Goffman, 1975, explore les mécanismes d'imposition et de gestion du stigmate et analyse, en partant de la rencontre avec l'autre, ce qui contribue à faire vaciller les fondements du « self - care ». Quant à Bury ,1982, il approfondit le phénomène de rupture ou de bouleversement (biographical disruption) qui s'établit avec l'entrée dans la maladie qui dure, en mettant l'accent sur les structures de vie quotidienne et de formes de connaissance qui y sont associées .

Parmi les éléments explicatifs de ce bouleversement figurent le fait que le patient doit, en premier lieu, faire face à un bouleversement des conduites. Cette première remise en 
question provoquée chez l'acteur et enfin la réponse à ce bouleversement sont liées à la mobilisation, par l'acteur, des ressources mises à sa disposition.

Cette conceptualisation de la rupture liée à la maladie chronique sera notre point de départ pour la discussion des résultats qui vont suivre.

Il s'agit donc, en référence à une recherche réalisée en Tunisie sur la construction socio culturelle du cancer, d'approfondir l'un des premiers segments de cette trajectoire, celui de l'annonce du diagnostic, initialement présenté par l'intermédiaire de cette recherche conduite selon la méthode de la théorisation ancrée.(Tinsa, 2003) .

Ainsi au niveau du présent texte, les significations existentielles de cette étape initiatrice de l'entrée dans la maladie seront appréhendées à partir de différentes stratégies déployées par les cliniciens et reçues par les patients et le questionnement inclura l'influence de l'activité institutionnelle.

C'est donc en référence à quelques résultats en rapport avec les stratégies d'annonce ,le contexte culturel entourant l'action et les attentes spécifiques dégagées des propos qu'ont bien voulu nous livrer des malades au prise avec l'expérience de la maladie et quelques uns de leurs accompagnateurs ( professionnels et familiaux) que nous reviendrons vers ces différents degrés de conscience de ces individus au prise avec l'expérience de la maladie. Notre intention sera de poursuivre ce questionnement sur leur capacité à se positionner sur ce continuum et à envisager les premiers pas à franchir dans une situation qui les renvoie obligatoirement vers l'adoption d'un nouveau rôle imposé par leur état de santé, les conduisant vers une remise en cause de leur identité et de tous les autres rôles qui les avaient, jusque là , maintenus au contact des autres .

Notre objectif est donc de prendre en compte les motivations des praticiens et le sens qu'ils accordent à l'étape de l'annonce du diagnostic et de les recontextualiser en référence à l'encadrement prévu par l'instance normative pendant la période d'hospitalisation .

Par ailleurs, la présentation de conditions spécifiques au contexte organisationnel devrait contribuer à identifier d'autres freins à l'ouverture afin de saisir comment l'organisation hospitalière intervient sur le destin social des patients en interrogeant sa contribution à la transformation de son identité.

\section{Aspects méthodologiques}

Cette analyse s'appuie sur un travail de terrain de 8 mois au sein principalement d'un service de chimiothérapie d'un Établissement Public de Santé de la ville de Tunis , spécialisé en oncologie - sur la base d'observations directes et d'entretiens informels puis semi-directifs auprès de patients soumis à une cure chimiothérapique . D' autres entretiens auprès de différents membres des équipes de soins (plus précisément des oncologues, une spécialiste du traitement de la douleur, des infirmiers et des membres impliqués dans le processus de gestion à l' exemple du directeur général et de la chef de service des malades . En complément des données collectées en milieu hospitalier, 
d'autres matériaux ont été apportés par l' intermédiaire de contacts établis en milieux associatifs auprès de personnes ressources actives dans ce milieu et de d'autres patients, logés dans une résidence annexe à l'hôpital ( mise sur pied par une structure associative , pour offrir une prise en charge gratuite à des patients issus de mieux socio économiques défavorisés et dont le domicile se trouve éloigné du centre de traitement ) durant des cures programmées en ambulatoire .

L'interactionnisme symbolique est choisi comme cadre de référence pour sa capacité à souligner la nature symbolique de la vie sociale et à insister sur le fait que

'les significations doivent être produites par les activités inter-agissantes des acteurs' .

(Blumer, 1969:5).

Ce cadre théorique sert d'ancrage à l'observation minutieuse du contexte pénétrant et influençant le parcours de chaque patient, initié par la découverte ou l'annonce de la maladie cancéreuse.

Quant à l'analyse des données collectées, elle a été effectuée à partir d'une méthode de théorisation ancrée et d'induction analytique. Rappelons que la théorisation ancrée consiste à développer, à comparer et à confronter les différentes hypothèses générées par l'analyse des différentes données collectées à partir du point de vue des différents informateurs et du travail d'observation, progressivement, au fur et à mesure que le travail de terrain se déroule. Cette méthode a donc été choisie pour son apport spécifique à l'analyse des dynamiques entourant l'entrée dans la maladie cancéreuse et sa capacité à faire ressortir les conditions favorisant les différentes stratégies d' annonce et les conséquences que cette étape, abordée selon son degré d' ouverture, peut avoir sur les étapes à venir et par conséquent, sur les différents segments des principales trajectoires.

Quant à l'induction analytique, elle nous a permis de nuancer et de raffiner le travail de conceptualisation. Elle se base sur une méthode d'analyse continue et vise à faire ressortir, tour à tour, les similitudes et les différents contrastes entre les données, ceci avec l'intention de retrouver les caractéristiques, les relations aussi bien que les déterminants des différentes variations au niveau de l'ouverture des différents contextes de conscience reconstitués à partir du procédé inductif.

Concernant les caractéristiques de l'échantillon sélectif, dernière étape de la démarche d'échantillonnage proposé en théorisation ancrée, il réunit parmi les informateurs principaux 29 patients et 4 membres de la famille. Les patients sont agés de 21 à62 ans avec un âge moyen de 42.85 ans, pour les hommes et de 48 ans pour les femmes .

Atteints de différentes formes de cancer, ils sont tous hospitalisés, au moment de 1' étude, pour une cure chimiothérapique. Cette technique d'échantillonnage sélectif est elle-même précédée d' un échantillonnage ouvert, visant la découverte des catégories analytiques devant orienter vers une meilleure compréhension du phénomène étudié, l' annonce du diagnostic et d'un échantillonnage axial, d'avantage systématisé pour 
permettre de couvrir systématiquement l'ensemble des sous-groupes et des situations s' offrant à notre observation et fortuite, pour profiter de toute nouvelle situation pouvant émerger du travail empirique initialement entrepris au moment de la définition et de la délimitation de la population étudiée.

D'autres informateurs que l'on qualifie de contextuels réunissent différents spécialistes et plus précisément les cancérologues, impliqués dans l'annonce de la maladie, des bénévoles et quelques cadres administratifs.

\section{Résultats}

Le schéma précédent tente de représenter la variation de la démarche d'annonce du diagnostic. Il met en relief un ensemble de conditions structurelles spécifiques au contexte appréhendé par l'intermédiaire du travail empirique ainsi que celles, sous diverses formes de combinaison et selon les acteurs observés, qui entourent et par conséquent influent sur la manière de dire ou de ne pas dire.

Nous nous limiterons, dans la partie qui va suivre, à une brève description des différents contextes de conscience façonnant l'annonce du diagnostic ainsi qu'à la présentation illustrative des stratégies préférentielles des acteurs, principalement les cliniciens, puisqu' ils se chargent, le plus souvent, de cette annonce du diagnostic .

\section{Le contexte ouvert et le diagnostic de la maladie}

\section{Le contexte ouvert progressivement}

Ce contexte ouvert, de manière progressive, lorsqu'il s'agit du diagnostic, découle du choix préférentiel de la majorité des spécialistes rencontrés durant la phase exploratoire . Fondé sur des valeurs liées au bien-être des patients, l'analyse de ce contexte nous conduit , bien que partiellement, au sens visé par cet acte d' annonce pour le clinicien, celui de préserver un sentiment $d$ ' espoir, en laissant, du moins au début, le patient à l'abri d'une certaine vérité liée à l' incertitude, à la chronicité et à l' incurabilité. Il protège également le professionnel en reportant une activité qui déclenche obligatoirement un sentiment d'inconfort lorsqu' on se trouve confronté à la souffrance de l'autre , l'exercice de sa pratique médicale étant lui-même au cœur d' une vision sotériologique . En appréhendant ainsi la nature de la souffrance physique mais aussi morale, ces cliniciens rappellent que la médecine parvient à unir les domaines du matériel et celui de l'éthique , en allant bien au-delà de la matérialité et des sciences de la nature sur lesquels pourtant elle repose.( Good,1994). 
La stratégie préférentielle, selon les propos collectés par l'intermédiaire des médecins en exercice ,est celle de l'ouverture progressive . S'ils rappellent qu'aucun schéma ne peut être tracé à l' avance et que chaque message s'ajuste au fil des rencontres, il est évident que l'on opte pour une ouverture progressive :

'il faut agir avec nos malades d' une manière très nuancée, on prend un peu plus de temps pour annoncer le diagnostic, c' est une révélation progressive, on parle de la maladie, de l'extension de cette maladie, des possibilités thérapeutiques, pour essayer de prononcer le mot cancer, ce n est pas une éventualité très grande chez-nous "(médecin clinicien, spécialisé en oncologie).

Cette ouverture progressive s'explique, selon plusieurs cliniciens, par son influence sur le maintien de l'espoir :

'on ne dit pas tout, on lui laisse une marge d' espoir, ce n' est pas un silence, $c$ ' est une manière douce d' annoncer des nouvelles difficiles,...." (médecin clinicien, spécialisé en oncologie)

Et toujours selon les spécialistes, cette stratégie convient aux besoins et difficultés des patients :

"au début et bien les malades ont leur propre système de défense. Bon, je ne suis pas spécialiste dans le domaine de la psychologie, mais en fait, tant quion est malade, on nie la maladie, on ne veut plus en parler..."

"les malades ne posent pas trop de questions, le malade a envie de se protéger, déjà il a besoin de digérer ce qu'il a appris, quand cela sera fait, il se décidera à poser de nouvelles questions..... :

\section{- Le contexte ouvert d'emblée}

Il s'agit, pour ce contexte bien particulier de patients mis au courant, presque accidentellement et même plutôt maladroitement, de leur maladie. Son ouverture soudaine et sans préparation est le résultat d' un manque de coordination entre les intervenants, de l' initiative d'un soignant qui prend la décision de dire, sans prise en compte du rôle qui est d'avantage imparti au médecin traitant.

On y retrouve également des sujets instruits dont certains exercent ou ont exercé une profession orientée vers le domaine de la santé. Dans ce cas, les questions précises, directes et pertinentes laissent peu de marge pour éloigner ou retarder la vérité à propos du type de cancer, du stade de la maladie et parfois même du pronostic vital.

\section{Le contexte fermé}


Ce contexte revêt, d'après les observations, deux variations : un contexte fermé en attente d'un diagnostic (on se trouve en présence de données insuffisantes pour valider le diagnostic) et un contexte maintenu fermé après la validation du diagnostic médical, à la demande spécifique de la famille, ou selon la stratégie d'ouverture sélectionnée et entretenu par le médecin traitant.

\section{- Le contexte fermé ou légèrement entrouvert, en attendant la confirmation du diagnostic}

Ce contexte est celui qui accompagne le patient durant toute la période d'investigation. Si certains patients vivent cette période avec l'espoir de voir leurs doutes et leurs craintes s'estomper du fait de la confirmation d'un état de non malignité, d'autres sombrent dans un état d'abattement ou expriment des sentiments de colère à l'égard de leur entourage et plus particulièrement envers l'institution médicale.

\section{- Le contexte maintenu fermé à la demande de la famille}

Ce contexte maintient le patient à l'écart de son expérience de la maladie, après la confirmation du diagnostic. Il répond à la demande d'une famille ou d'un membre en particulier. On recommande à l'équipe médicale, dans ces circonstances, de ne pas révéler le diagnostic médical au patient :

Voici l'exemple d'un jeune homme de 36ans qui s'est trouvé mis à l'écart de son diagnostic, privé d'information concernant sa thérapeutique et son pronostic. Les propos qui vont suivre ont été exprimés par sa sœur qui est la première personne à avoir fait barrière à l'information :

\footnotetext{
'mon frère n'est pas au courant de son diagnostic, il pense qu' il s' agit d' un simple kyste qui doit réduire sous l' effet de la radiothérapie, et de la chimiothérapie, $j$ ai menti à mon frère, je lui ai $m^{\wedge}$ me dit qu' on fait de la radiothérapie pour des enfants qui ont des végétations, on doit mentir car sinon ce serait la catastrophe pour lui, il n y a que moi et mon mari qui sommes au courant....."
}

Trois conditions identifiées au cours de l'étude contribuent à la compréhension de ce contexte fermé. Mises en évidence par l'intermédiaire de différents témoignages, ces conditions distinctement répertoriés éclairent sur les motifs de fermeture et sur le pourquoi de la mise à l' écart du patient. Elles correspondent à l'évaluation de ce qui ne doit pas être entendu ou porté à sa connaissance. Elles impliquent la reconnaissance d'un droit de prendre cette décision de tenir l'autre à l' écart de son vécu et enfin elles supposent l'acceptation, en tant que professionnel, d'entrer dans ce scénario dicté , imaginé et improvisé par un membre de la famille.

D'autres conditions s'opposent aux précédentes et pourraient interférer avec celles- 
ci et changer le cours même des interactions .Elles relèvent d'une prise de position ferme de certains spécialistes qui prend en compte leur propre pratique et leur capacité réflexive:
'pour moi, cela a beaucoup évolué, d'une intuition qui était fausse, oui mon intuition s'est avérée fausse, je pensais que la maladie était tellement terrifiante au début de ma pratique, oui je pensais que personne ne pouvait en parler et se parler et maintenant je souhaiterais dire la vérité sauf demande expresse du malade de ne rien lui dire... ."(médecin clinicien spécialisé en oncologie ).

\section{Le contexte feint}

Contexte difficile à identifier, reconstruit à partir des propos des partenaires (professionnels et patients) et des données collectées au moyen de l'observation participante. Il réunit différents patients mis au courant du diagnostic médical, qui demandent expressément au médecin traitant de ne rien dire à la famille, afin de les rassurer. Dans la plupart des situations observées, on remarque que la famille, le plus souvent au courant de la vérité, feint à son tour de ne pas savoir, pour ne pas aller à l'encontre de la demande du patient. Commence alors une situation qui laisse les accompagnateurs et les proches à distance du patient, lequel ne peut verbaliser son vécu et exprimer ses souffrances et inquiétudes en présence de son entourage.

Ces quelques résultats mettent en relief différentes stratégies douverture de la connaissance telle que la stratégie préférentielle qui consiste à opter pour une vérité révélée avec prudence, dans la progression, l'étalant selon les signaux et attitudes dévoilées par le patient sur différentes rencontres, l'intention première étant,pour différents informateurs, de ne pas décourager, traumatiser en choisissant d' ajuster son discours de manière à laisser au patient, le temps d'apprivoiser une situation qui l'ouvre sur un nouveau monde.

C'est ainsi que différents axes de comparaison résultant de l'insertion d'éléments en rapport avec le vécu de la maladie, selon les contextes explicatifs, contribuent à une compréhension plus approfondie de différents phénomènes signifiant la maladie cancéreuse .

L'un des principaux axes intitulés degré d'ouverture et de fermeture du contexte de conscience, à propos du diagnostic de la maladie illustre bien les quelques trajectoires jalonnant le parcours des patients rencontrés. Il associe différents éléments contextuels (valeurs curatives et humanistes des médecins, philosophie et mission actuelle de l'Institut de Cancérologie, mentalité du clinicien, symboles -clé liés à la maladie, espoir de vivre des malades et préservation de cet espoir.....) à l'aspect dynamique de ce champ de conscience et laisse entrevoir les conséquences de l'agir sur le vécu et le niveau d'implication du patient sur sa propre expérience. 
Une attention particulière portée à l'agir du sujet et aux conditions orientant ces actions guide notre démarche. Différents évènements qui indiquent une distance entretenue entre profanes et savants et une forme de silence destiné, semble t'il, à protéger celui qui ne doit pas savoir, servent de point de comparaison à d'autres situations qui mettent en évidence une recherche de la vérité, différemment exprimé d'un sujet à l'autre et d'un moment à l'autre, selon chaque parcours individuel. En effet, s' est autour de cette confrontation du malade et des professionnels de santé à une réalité plus ou moins clarifiée ou plus ou moins prête à être envisagée et de différents modes d'interaction à partir de l'observation et des discours collectés auprès des acteurs que l'on commence à reconstruire les contextes de conscience.

Ces différents contextes de conscience se réfèrent donc, selon Glaser et Strauss, 1966, aux structures et processus impliqués dans la gestion du savoir relatif au déroulement de la maladie, tout le long de sa trajectoire, de la phase diagnostic à sa guérison ou à celle de sa fin de vie.

Ils nous aident à appréhender ou à saisir analytiquement les situations multiples repérées au cours de la phase exploratoire et à déboucher sur différentes images distinctes mais apparentées, construites à partir de ce que chaque personne interagissant avec celle vivant cette expérience de santé sait du statut défini de celle ci .À cette connaissance s' ajoute l'évaluation de l'information pouvant être dévoilée aussi bien que l'intention d'ouverture du champ de la connaissance concernant son diagnostic par différents acteurs impliqués dans ce processus de gestion de la maladie.

\section{Discussion}

La relation médecin-malade a été décrite en référence aux différentes stratégies d'annonce du diagnostic influant sur le degré d'ouverture du contexte et par conséquent sur le niveau d'information transmis au patient à propos de son état de santé et cela avant, à l'occasion ou dans d'autres cas, après cette confirmation du diagnostic médical.

Les spécialistes de la cancérologie et plus particulièrement les oncologues observés et interrogés interviennent en combinant les tactiques suivantes ou tout simplement en se centrant plus particulièrement sur l'une d'elles.

Ces tactiques peuvent se résumer ainsi :

- attendre que le patient pose lui-même les questions

- lui poser indirectement les questions, afin de savoir ce qu'il sait déjà et avec l' intention de s' ajuster au mieux à son niveau de connaissance

- mentir au malade (à la demande de la famille) en lui présentant un autre diagnostic au caractère plus bénin, tel que celui du kyste hydatique, devant masquer , aux regard du patient, le cancer du poumon

- demeurer imprécis, en survolant le problème de santé 
- dire toute la vérité à ceux, qui ,selon les spécialistes, peuvent y faire face

- annoncer brusquement ou accidentellement la vérité, sans préparation ou sans progression.

Répertorier les stratégies préférentielles donne accès aux ressources de sens et à la capacité interprétative des principaux acteurs impliqués dans cette démarche d' annonce . S'intéresser à l' action et par conséquent à la manière dont les différents partenaires de la relation soignant-soigné se perçoivent mutuellement et prendre en compte les intentions qu'ils s'attribuent les uns, les autres ou s' interroger sur la rupture biographique et l'entrée du destinataire du message dans le monde de la maladie chronique n' exclut pas l'intérêt du regard posé sur l' environnement institutionnel et l' encadrement qu' il prévoit pour protéger les droits de savoir de celui qui ne sait pas encore ou du moins insuffisamment . En effet tous ces éléments interviennent, plus ou moins directement, dans le contexte de la biographie du malade, rendue discontinue par la maladie.

\section{Stratégies d'ouverture et ressources de sens}

Les stratégies préférentielles répertoriées en matière d'annonce de la maladie trouvent une diversité d'orientation qui, dans certaines situations, peut demander aux professionnels de taire la vérité à propos du diagnostic, sous évaluant de ce fait le rôle du parent et sa capacité à franchir lui-même les étapes d'une longue trajectoire qui exige sa participation éclairée. Projeté de plein pied dans ce processus de mobilisation familiale, supportée par la pensée, en tant que processus symbolique, elle intervient pour transformer cette étape d'entrée dans la maladie en une matière première de sens et décision pesée, évaluée, réfléchie pour le bien du malade et le confort de l' accompagnateur (Thomas W,2000)

Les actions sont posées en prévision du comportement du patient. En effet, si on réduit l'information, jusqu' à dans certains cas, taire le diagnostic et contribuer à la fermeture du contexte de conscience ) c' est avec l'intention de maintenir un sentiment d' espoir ( Saillant, 1988,Patterson,1987,Holland,1987 et 1989,Schaffer et Lind,1990, Townsend,1978) .

La précaution de se protéger de ce climat chargé d'incertitude, dans un environnement renouvelant et valorisant des pratiques inspirées du paradigme technico-scientifique, renforce l'entité médicale. Moins affirmée, la culture soignante, malgré les nombreux besoins de soutenir et d'entretenir la vie, durant le déroulement des nombreuses trajectoires de la maladie, à l'exemple des étapes d'administration de la thérapeutique sélectionnée par les cliniciens, confirme une médecine centrée sur le curatif, l' organe, la lésion, la maladie "disease".

L'interaction semble bien orientée par la capacité réflexive du professionnel de la santé et sa disposition à se mettre à la place du patient pour parvenir à une meilleure compréhension. 
Et ce travail interprétatif exigent de l'acteur un retour vers ses références sociales et culturelles et se sert de significations plus ou moins partagées par les membres de différents groupe.C'est ainsi que dans ce pays du Maghreb, tout comme dans différents pays méditerranées le cancer continue de véhiculer des images de mort , d'incurabilité, de souffrance et de détérioration de la personne.( Holland,1987 et 1989).

Par ailleurs ,les individus rencontrés n'appartiennent pas nécessairement aux mêmes mondes sociaux et les soignants s' appliquent à nuancer leurs approches en fonction du niveau culturel de l'individu, de la profession, de l'âge , du sexe, du type de cancer et du stade de la maladie .( Pratt L. et al. ,1957 ; Davis F. 1963 ....) ou de l'anticipation d'une réaction émotionnelle non favorable ( Fitts et Ravdlin,1953 cité par J. Mc.Intosh,1974)

Toutes ces précautions ne mettent pas les protagonistes à l'abri de tensions, de malaises , qui peuvent, dans certaines situations, lorsque le patient ne s' accorde pas le droit d'intervenir verbalement pour demander des clarifications ou pour exprimer son malaise, conduire les partenaires de la relation thérapeutique dans de réels conflits d' interprétation. C'est ainsi qu'une situation qui idéalement ne devrait cesser de se redéfinir, pour s'ajuster progressivement par l'intermédiaire de ce partage de sens , peut se trouver figer, en raison du manque ou de l'absence de rétroaction. Ce qui ne peut servir qu'à alimenter l'écart entre l'individu et cette expérience de vie et par conséquent , bien que les motivations premières puisaient dans les valeurs éthiques du praticien , interférer avec tout ce travail de gestion de la maladie ( Strauss ,et Glaser,1968,Strauss ,Fagerhaugh, Suczek and Wiener,1982 Strauss et Corbin,1988,........)

Ce modèle d'interaction, observé autour de l'annonce du diagnostic, fournit un bon point de départ pour étudier ce qui se passe quand deux personnes parlent et agissent face à face ( Strauss,1992:59)

Ce temps d'arrêt fixé sur le déroulement de l'interaction à l'intérieur d'un micro contexte hospitalier, au moment de l'annonce du diagnostic, rejoint les explications fournies par Le Breton, 2004: 51 , comme quoi elles ne sont pas des processus mécaniques se greffant sur des statuts et des rôles. Ainsi bien que le cadre formel oriente le sens de l'action, les partenaires privilégient l'ajustement à partir de tout un travail interprétatif et semblent résister à toute "actualisation mécanique d'une conformité" ( Le Breton D. 2004 : 51 )

Cette conception interactionniste de la conscience développée par Strauss éclaire notre compréhension des logiques sociales qui orientent plus particulièrement le sens de certaines interactions. Certaines situations s'ajustent aux attentes du patient, d' autres , au contraire, auront à subir un contexte délimité avec une intention première de protéger et de nourrir un sentiment d'espoir . D' autres, moins fréquentes, se trouvent ajustées aux demandes incessantes de famille, telle que la sœur aînée, elle-même professionnelle de santé qui demanda de ne pas dire, pour le moment, sous évaluant la capacité du parent à faire face et cherchant, en tant que proche, à se protéger d' une situation difficile également pour 1 entourage qui $n$ est pas nécessairement bien préparé à ce changement . Ainsi, dans cette étude, le degré d'aliénation du patient, conséquence 
directe de la fermeture du certains contextes de conscience, aura une influence directe sur la perception d' un manque de pouvoir et donc sur la représentation, pour le patient, d' une incapacité à influencer, par son comportement, le résultat de différents évènements qui entravent sa vie.(Skipper J.K.,1965,Sieman et Evan, cité dans Mc.Intosh,1974) .

\section{Annonce du diagnostic et cadre institutionnel}

Les interactions nourries dans cette sphère spécifique au monde médical nécessitent différents ajustements en fonction du processus d'interprétation. Différentes conditions entourent l'action et semblent influer sur le degré d'ouverture du contexte de conscience de l'annonce du diagnostic. Parmi celles présentées précédemment, on retrouve une forte présence du stigmate de la mort qui continue de frapper l'univers de la cancérologie en Tunisie, malgré d' importants investissements au niveau de la sensibilisation et de 1 information du citoyen, des protocoles thérapeutiques adressés à des patients dont les trajectoires s'annoncent dominées par de nombreuses incertitudes, l'adhésion des cliniciens à la mission de la médecine curative traditionnelle qui pourrait être altérée si le patient vivait une perte d'espoir.

D' autres conditions, tel que le temps mis à la disposition des soignants pour une consultation d' annonce, les frontières qui semblent délimiter et isoler les différentes catégories professionnelles ou encore l' organisation spaciale des locaux interferent ,d'après les témoignages et les observations empiriques, dans la conduite des échanges et parviennent, dans différentes circonstances, à imposer des stratégies qui correspondent peu à la conception des acteurs de leur rôle et de la manière de l' assurer .

C'est en cherchant à relier les compétences des professionnels de la santé impliqués dans l'annonce du diagnostic à différents éléments structurels que l'on peut entrevoir certaines dimensions de la dualité du structurel dans l'interaction .(Guiddens ,1987:78)

En tentant de présenter quelques facettes de l'interaction, on se retrouve donc en présence de cette interpénétration de la signification,, selon le schème d'interprétation et de la légitimation selon l'orientation des normes de pratique peu officialisées, en ce qui a trait, entre autre, au déroulement des consultations d' annonce. S'y trouve mis en évidence ces réservoirs utilisés de façon réflexive par les différents partenaires, pour produire et reproduire différentes formes d'interaction. (Guiddens,1987:. 78 )

En effet, utiliser les réservoirs de connaissance de façon réflexive dans la production et la reproduction d'interactions pour décider du degré d'ouverture du contexte d' annonce ou tenter de rationaliser sa démarche d' ouverture en évaluant la compétence de l'autre à faire face à la vérité n' exclut pas d' autres éléments aussi importants de cette rationalisation de l'action .

Ce questionnement autour de cette dimension normative suggère donc un déplacement du côté de l'institué pour examiner les règles et les normes contributives à l'encadrement des professionnels en matière d'annonce du diagnostic autour de cette 
étape de la trajectoire. Il semble en effet difficile de parler de segments de trajectoire d' annonce dans la mesure ou les intervenants en poste ne sont pas parvenus à instituer une régulation commune .Culture organisationnelle centrée sur le non dit, stigmate de la mort, prédominances des valeurs curatives et des approches technicistes, division du travail déployée autour de l'administration des cures devraient inciter les protagonistes en interaction au moment de l'annonce à circonscrire leur champ d intervention et à officialiser les différentes contributions.

La création d'un monde social du traitement curatif du cancer (Becker et Strauss ) renvoie implicitement à des réseaux de partenaires d' univers différents et de chaîne de coopérations et de complémentarité. Sa dynamique de constitution est directement liée au travail de théorisation de la discipline ( Strauss,1984:176-177) et le rôle et les activités de chaque catégorie d' acteurs gagneraient à être dégagés, harmonisés et spécifiés en fonction de la philosophie d'annonce qui vise implicitement la protection de l'intégrité de la personne et un accompagnement réconfortant. Ainsi la constitution d'un monde social sert de point d'ancrage pour autonomiser et consolider une pratique orientée vers l'annonce du diagnostic et transformer une étape en un segment de trajectoire parcouru par un être réflexif capable de monitorer son expérience et de donner les raisons de ses interventions.

L'aspiration d'établir une relation de proximité avec le patient au moment de son entrée dans la maladie et l'affirmation d'une responsabilité d'accompagnement sont ainsi conçues comme d'autres compétences spécifiques à promouvoir, à entretenir et par conséquent à institutionnaliser dans ce monde de la cancérologie.

\section{Constitution de l'identité du malade, rupture biographique et entrée dans le monde de la maladie}

En s'efforçant de maîtriser le désordre provoqué par une maladie qui se caractérise par sa gravité, dépendamment, entre autre, du site de l'atteinte et de l'état d'avancement au moment de sa découverte, les professionnels de la santé prennent en compte le choc émotionnel qui sera provoqué au moment de la révélation de la maladie et tente, dans la plupart des cas, de procéder d'une manière progressive, (ouverture progressive du contexte de conscience).

La personne cependant n'apparaît pas vraiment au centre de la démarche de travail et les valeurs curatives semblent conduire et soutenir avec force les pratiques professionnelles. Guérir, prolonger la vie, traiter la maladie deviennent la mission première de l'unité et on se centre d'avantage sur la pathologie et le corps et , malgré l'intentionnalité des praticiens de protéger et de soutenir le déroulement des tâches, la coordination de certaines informations consignées au dossier médical sont en rapport avec la thérapeutique et peu favorable à la reconnaissance de la personne comme sujet 
,non réductible à une pathologie ou à un corps humain.

Ainsi cette dernière partie de la discussion, en revenant sur les modes d'interaction professionnels et patients par l' intermédiaire des différents contextes de conscience répertoriés, nous aide à saisir comment l'organisation intervient sur le destin social de l' individu en contribuant, malgré une restriction de l'information, à engager un processus de transformation de son identité sociale.

Cette phase d'admission ne peut être appréhendée comme un segment de la trajectoire d'annonce du fait de la difficulté à instituer une régulation commune. Son mode de gestion apparaît peu favorable à l'esprit de collaboration entre les différentes disciplines et, ,par conséquent, au renforcement de la complémentarité entre les différentes équipes et spécialités.

Cette étape d'annonce doit être considérée comme un moment déterminant de la carrière du malade. Elle marque le passage vers un nouveau statut, tout en tentant d' apprécier le niveau de conscience du patient à l' égard de sa maladie et en évaluant sa capacité à recevoir une information plus détaillée. Cette évaluation se base, entre autre, sur des caractéristiques socio- professionnelles (niveau d' instruction, profession, statut social, ....) et les professionnels s' efforcent de s' adapter aux contraintes de la structure actuelle ( brièveté du temps accorder à chaque consultation, ouverture fréquente des portes de bureau, partage des espaces entre confrères,,,,,), comme différentes conditions largement discutées précédemment.

Si les consultations apparaissent pour chaque professionnel comme des changements de statuts organisés ( Strauss 1992:.), pour lesquels il est presque nécessaire de ménager une période d' accoutumance qui suivra l' entrée officielle dans de nouveaux statuts ( Strauss, 1992: 111),cette intention partagée par la plupart des cliniciens rencontrés est difficilement coordonnable entre les différents intervenants impliqués dans la consultation d' annonce ( médecin, psychologue, soignant...) du fait de l'absence de règles institutionnalisés. Mais on constate que de nombreuses procédures employées dans le service d'oncologie , au jour le jour, ont une influence marquante sur les pratiques professionnelles.

Toutefois la rupture biographique ne peut être épargné quelque soit l' état d' avancement, le type de cancer et les chances de guérison. Cette rupture est d' autant plus difficile quelle commence par des rencontres beaucoup plus régulées dans une intention protectrice et celle de faire face aux contraintes de temps que par une organisation des pratiques qui considèrent le patient comme un partenaire du contrat. Le patient commence dans sa carrière morale de malade atteint d'un cancer (Goffman, 1994:56 ) marqué par de fortes périodes d'incertitude pouvant être accentuée par une limitation de l'information et par conséquent une forme de distanciation de sa propre expérience.

Certaines conséquences immédiates telle que l'anxiété, un stress émotionnel important, un état d'abattement ...témoignent de cette rupture biographique qu'on souhaitait pourtant éviter ou du moins alléger ( Bury ,1982:167-182) 
D'autres, plus lointaines, pénétreront le déroulement du prochain segment de la trajectoire à savoir la démarche thérapeutique et mis à part tout l' inconfort sur le plan émotionnel et moral, pourront interférer avec la démarche thérapeutique et avoir , selon les témoignages, des liens directs avec des interruptions momentanées ou définitives du suivi thérapeutique.

Autre conséquence sur le déroulement des prochaines étapes ou segments de trajectoires initiés par une consultation d'annonce peu propice à une construction conjointe du sens de l'hospitalisation est la reproduction de rencontres dirigistes pour le bien du malade. En les éloignant d'un travail de contractualisation de la relation au soin et à la démarche thérapeutique, pré-requis à l'intégration d'attitudes responsables dans la gestion de sa maladie, elle ne favorise pas la conformité ??de l'individu à son assignation statutaire à partir de laquelle il pourrait s' initier à ce long travail de gestion de la maladie portant, notamment, sur le soulagement de symptômes liés à la chimiothérapie et plus encore sur sa nouvelle expérience de vie et par conséquent sur la conception de son identité en mouvement.

Mais comment ' instituer,' assigner une essence, une compétence, imposer un droit d' être qui est un devoir être" et comment 'signifier à quelqu'un ce qu' il est et lui signifier qu' il a à se conduire en conséquence "( Bourdieu,1986:126) lorsqu' on ne parvient pas circonscrire les étapes de cette consultation et à tenter un travail de collaboration entre les membres d'une équipe pluridisciplinaire?

\section{Conclusion}

Réinterroger la notion de rupture biographique en se référant à un point de bifurcation de la carrière du malade, celui de la confirmation du diagnostic ou de bouleversement du à l'émergence de la maladie ( Bury:1982: 69 ) rappelle que l'entrée dans la maladie se déplace de la phase initiale interrogée sous l'angle de symptômes et de signes cliniques par les spécialistes de la clinique vers une succession de positions occupées par chaque acteur et distinctives entre les individus.

Faites de sorties de rôles et de création de d'autres rôles, de travail de mise en cohérence( Ricoeur,1990) dans un climat lourdement chargé d' incertitude et de symbolique morbide, cette rupture renvoie également vers l' acceptation du statut du bon malade ou le déplacement de tactiques pour parvenir à s' accorder, non sans difficultés et souffrance morale, à cette situation au niveau du soi afin de parvenir à assurer la continuité identitaire. Entendue comme point de bifurcation, de manière plus ou moins totale, plus ou moins institutionnalisée, plus ou moins conscientisée , cette rupture constitue, en effet, un moment décisif, qui se révèle par un incident dans la conception de soi.

Différentes conditions structurelles venant de la maladie chronique, de la biographie du patient et de sa vie quotidienne pèsent sur le travail de gestion de la maladie 
(Strauss 1968,1978,1982,1988...) et contribuent à déterminer les points de rupture qui assombrissent les trajectoires .

D'autres éléments, venant du contexte immédiat, entourent plus directement l' action et interviennent également de manière à influencer le cours des interactions. D' abord les 'frontières spatio-temporelles "Giddens, 1986 ) permettent de circonscrire les parcelles d'interaction, lesquelles sont imprégnées de "marqueurs" symboliques ou physiques, ensuite la co-présence des partenaires de la relation thérapeutique et enfin le degré d'implication possible pour chaque partenaire de cette relation.

Cette dimension serait apparentée selon Giddens, 1986, à une sorte de

'vision circulaire de la construction du monde social ou ses dimensions structurantes se situant à la fois avant l'action, comme ses conditions et après, comme des produits de celles-ci”.

Rappelons ici que le point de départ de cet article était les différents acteurs impliqués plus ou moins directement dans les stratégies d'annonce du diagnostic. Agissant et interagissant en fonction du sens qu'ils attribuent à leur agir et à celui des autres, ils portent tous un univers symbolique de valeurs et d'idéologies qu'ils entretiennent et, selon les circonstances et les spécificités du moment, tentent de modifier et parfois de reconstruire au sein de différentes institutions qui encadrent l'expérience de la maladie.

Modernisation des structures, difficultés de régulation des activités liées à la mission de l' institution et aussi bien qu' à celle de l'unité d' oncologie observée et intention de démocratisation des droits des patients parviennent à tisser des liens inégalement resserrés entre les partenaires de la relation thérapeutique. Cependant que l'on se réfère aux propos de patients contestant la restriction de l'information en rapport avec leur état de santé et évoquant les répercussions négatives sur leur propre parcours ou que l' on s'arrête sur celui des praticiens plus directement impliqués dans le développement de la discipline et le renouvellement de pratiques institutionnelles, on s' interroge sur l' apport de la formalisation des explications concernant le dispositif d' annonce du diagnostic . Peut elle offrir des éléments de réponse aux inégalités dans la manière de vivre une situation de rencontre plus ou moins partagée, selon le parcours du patient et par conséquent lui assurer une meilleure mobilité au niveau des différents segments de la trajectoire?

S' inscrivant au sein de structures au mode de fonctionnement peu formalisé en ce qui concerne les droits à l' information, les formalités d' accueil, le consentement éclairé, pour ne citer que ces exemples et par conséquent plus portées à nourrir des accords tacites entre les professionnels et un ajustement des" stratégies $d$ ' annonce au cas pas cas "( clinicien spécialisé en oncologie) , on remarque, entre autre, que certaines conditions relatives au profil des patients peuvent influer sur les stratégies d' annonce , pensées et initiés, le plus souvent pas les cliniciens. Ce qui permet de dire que le 
niveau élevé d'instruction, une profession apparentée avec le domaine de la médecine, ou encore l'appartenance à une classe sociale plus favorisée, seraient des facteurs favorables à une transmission plus dense de l'information et par conséquent à un cheminement mieux éclairé.

Ces différentes stratégies d'annonce d'une maladie grave, en apparaissant 'intrinsèques à la compétence" des patients reposent ici toute la question de la légitimité des rapports sociaux face aux contraintes de la situation, comme celle d'évaluer la capacité de savoir en partant des représentations des professionnels, des défaillances au niveau du temps de rencontre qui leur est octroyé pour dire et dire suffisamment ou encore de la disposition et de l'aménagement des espaces très souvent ouverts, achalandés, allant jusqu'à réunir différents postes de travail dans un même lieu de consultation, à l'exemple d' une consultation spécifique dans le traitement de la douleur devant partager son espace entre deux médecins spécialistes.

Ce défi de démocratisation des droits du patient, en s'appuyant sur les stratégies d' annonce du diagnostic d' une maladie qui présente une dimension sociale facilement identifiable, nous apparaît étroitement lié au contrôle réflexif des différents partenaires ,lequel ne peut cependant pas se dissocier du cadre de l'interaction.

En effets les nombreuses formes d'interaction contribuent à façonner les différents contextes de conscience qui s'initient dès que l'on soupçonne la présence de la maladie .Pouvant être regroupées selon différents degré d'ouverture-fermeture, en fonction des différentes stratégies d'annonce, pénétrées et par conséquent influencées par les différentes conditions entourant les rencontres, elles alimentent un important questionnement sur les "conséquences non intentionnelles" (Giddens, 1986 ) pouvant interférer plus ou moins directement sur la démarche thérapeutique et sur le pronostic vital de la personne (arrêt du traitement décidé par le patient, discontinuité ou interruption des consultations de contrôle, recours à un guérisseur ..... ).

Toutefois si la codification d'une certaine manière de dispenser des informations recevables et discutables en rapport avec l'annonce du diagnostic et l'entrée dans la phase thérapeutique semble favorable à une plus grande ouverture du contexte de conscience, quelque soit les pré-requis du bénéficiaire et ses capacités à saisir l'information, peuton supposer qu' elle est indispensable à l'accompagnement du patient au moment du changement initial de trajectoire?

Selon A. Giddens, institutionnaliser ces conduites à tenir et par conséquent délimiter les orientations de l'engagement normatif n'est pas le seul élément de la rationalisation de l'action et chaque agent( Giddens,1986, 1998), tout en mettant en œuvre des décisions qui lui agréent, a la capacité , quoi que inégalement, de mobiliser les biais inhérents aux institutions ( Bachrach et Baratz, 1962) .Il nous apparaît ainsi comme le principal moyen de préserver la liberté du patient, quelque soit le fonctionnement de la structure et son degré d' ajustement aux problématiques du moment et devient, de ce fait, la condition et le résultat d' activités entreprises par les partenaires de cette relation. 
Or cet apport informatif du professionnel semble inégalement régulé d'une situation à l'autre et d'un patient à l'autre .Des critères, tel que le niveau d'instruction, la profession exercée ou encore le statut socio-économique peuvent contribuer à nuancer la densité et le type d'information à transmettre et par conséquent sous estimer la capacité de comprendre de celui qui ne dispose que d'un faible niveau d'instruction et par conséquent négliger ou dévaloriser son implication. D'autres motifs de restriction de l'information, liés à la protection de l'intégrité et à la lutte contre le stress ou contre le sentiment de perdre espoir, sont des exemples discutés et illustrés précédemment.

.Cet apport est inégalement lié à la reconnaissance du rôle actif de l'individu dans ce changement de trajectoire et par conséquent à celle de son travail de gestion qui est la condition essentielle d'un" projet réflexif et négocié" (Giddens,1986: 92 et 91), compris comme réel soutien à la transformation d'identité de soi, passage obligé et amorcé au moment de l'entrée d'une maladie qui dure, qui peut récidiver et dont on ignore la durée et le nombre de segments à venir et à assumer. Ce qui suppose la reconnaissance des compétences et des capacités des différents acteurs d'agir malgré les contraintes des différents systèmes, inégalement perçue, en fonction de caractéristiques individuelles, notamment.

Par ailleurs, la prise en compte des conséquences non intentionnelles sur la santé de l' individu , pouvant aller jusqu' à l' assombrissement du pronostic vital ,en raison d' un manque d' information souligne l' intérêt d' entourer les interactions de conditions favorisant leur continuité et une certaine routinisation sans perdre de vue le fait qu' elles ne peuvent ni garantir les approches systématisées ,ni une annonce de la vérité d' une même intensité, quelque soit le profil et les attentes des sujets frappés par la maladie. C'est dans ce sens que nous pensons que les plans programmant le suivi et l'accompagnement des malades chroniques pourraient devenir des artifices, si on ne prend pas en compte ce qui entoure l' action et si l' on ne s' arrête pas sur les richesses et les spécificités de nos systèmes moins formalisés mais rappelant une flexibilité à explorer et à interpréter. L" orchestration des tâches, autour et à partir de l'acteur principalement concerné par cette rupture biographique, exige des activités en amont et un nouveau regard porté sur la structure hospitalière actuelle, très peu exploré, ainsi que sur l'expérience vécue par le sujet lui-même et bien souvent, dans le cas de maladies au long cours , par les proches et les personnes les plus significatives.

\section{Références bibliographiques}

Bachrach P. et Baratz M. 1962 .- The two faces of power in American Political

Science Review, vol.56

Becker H.-1985 Outsiders : Études de sociologie de la déviance .- Paris :

Éditions Métailié

Bourdieu P.1986 .- Ce que parler veut dire .- Paris : Fayard.- 245p 
Bury M.1982.- Chronic illness as biographic disruption in Sociology of Health and Illness vol. 4,no.2 ,juillet ,pp. 167-182

Charmaz K.1987.Struggling for a self : identity levels of the chronically ill.

In J. Roth and P.Conrad (eds), Research in the Sociology of Health Care : The Experience and Management of Chronic Illness . Greenwich : JAIPress.-pp.283-321

Davis F.,1963.- Passage Through Crisis .- Bobb-Merrill. - Fillieule O. (2001)

Propositions pour une analyse processuelle de l'engagement individuel", in Olivier Fillieule et Nonna Mayer, (dir) dossier "Devenirs Militants", Revue française de science politique, vol 51, n¹, 2001, p. 199-215

Fitts et Ravdlin,1953 cité dans McIntosh,1974.- Processes of Communication, Information Seeking and Control Associated with Cancer. -in Social Science and Medicine, 8, 1974, p. 167-187.

Foucault M.1987.- Naissance de la clinique.- Paris: P.U.F.,Coll. 'Galien".214p.

Fox R.1988.L' incertitude médicale .Paris :L'Harmattan, coll. 'Catalyses”.-202p

Le Breton D.2004.-L'interactionnisme symbolique, Paris, PUF, Quadrige

Giddens A .1986.La constitution de la société :éléments de de la théorie de la structuration .- Paris : PUF

Good B.J.1994.-Medicine,rationality and expérience: An anthropological perspective.- Cambridge University Press,239p. Le Breton D.

2004.-Linteractionnisme symbolique, Paris, PUF, Quadrige

Holland,J.C.,Geary N.A.and Tross S.1987.- An international survey of physician attitudes and practice in regard to revealing the diagnostic the diagnostic of cancer in Cancer Investigation 5.- pp.151-154

Holland,J.C.1989.- Now we tell-but how well? in Journal of Clinical Oncology 7 - - pp. 557-559

Kenneth H. et Tucker Jr. 1998.- Anthony Giddens and Modern Social Theory.London : Sage Publication

Le Breton D.2004.-L'interactionnisme symbolique, Paris, PUF, Quadrige,

McIntosh J .1974.- Processes of Communication ,Information Seeking and Control Associated with Cancer .- in Social Science and Medecine,8,.- pp. 167187

McIntosh J.1977.- Communication and Awareness in a Cancer Ward.- London: Croom Helm, New-York: Prodist

PattersonR.1987.- Public opinion on cancer in Lancet Oct. 23,857

Pratt L.et al1.1957 .- Physicians views on the level of medical information among patients .- in Am. J. Publ.Hich,no.47,1277

Ricoeur P. 1990.- Soi-même comme un autre, Le Seuil, Coll "Points Essais" Saillant F.1988).-Cancer et Culture.- Montréal:Éditions Saint-Martin.-318p. 
Sieman et Evan, cité dans Mc. Intosh ,op.cit.

Skipper J.K.,1965.- Social interaction and patient care .- London : J.B. Lippincott.pp. $167-179$

Strauss et Glaser.1968.- Time for dying.- Chicago: Adline; Weidenfeld et Nicholson

Strauss A.1978.- Négociations,varieties,contexts,processes and social order.- San Francisco, Jossey-Bass

Strauss A.,FagerhaughS.,Suczek B.and Wiener C.(1982).- The work of hospitalized patients in Social science and Medicine, vol.16.-pp.977-986

Strauss A.1982.-Social worlds and their legitimation processes in N.Denzin(éd), Studies in Symbolic Interaction,vol.5,Greenwich Londres ,JAI Press inc. - pp. 123-139

Strauss et Corbin.1988.- Unending work and care : managing chronic illness at home -- San Francisco : Jossey-Bass

Strauss,A.1992.- Miroirs et masques. Une introduction à l' interactionnisme .- Paris: Métailié.- 197p.

Thomas L.V.,2000.- Les chairs de la mort.-Paris: Sanofi- Synthélabo,coll.”Les empêcheurs de penser en rond".- 573P

Tinsa F.2003.- La construction socio-culturelle de la maladie cancéreuse en Tunisie, thèse sous la direction de R.Boukraa, Université de Tunis, Faculté des Sciences Humaines et Sociales de Tunis .-379 p et annexes 